\title{
Bachem - Insights into Innovative and Sustainable Peptide Chemistry and Technology by the Leading Independent Manufacturer of TIDES
}

\author{
Sandip Jadhav, Wolfgang Seufert, Carolin Lechner, and Ralph Schönleber ${ }^{\star}$
}

\begin{abstract}
Since its foundation in 1971, Bachem has grown sustainably over the last 50 years and is excellently positioned as the leading company for the development and production of TIDES i.e. peptides and oligonucleotides. Bachem's success relies on its commitment to manufacturing high-quality active pharmaceutical ingredients (APIs) alongside its continual passion for innovative chemistry and technologies. This review aims at summarizing improvements in high-quality peptide manufacturing as well as recent advances towards sustainable and innovative technology in peptide chemistry, thereby reducing the environmental footprint.
\end{abstract}

Keywords: Bachem · Molecular Hiving ${ }^{\mathrm{TM}} \cdot$ Peptide APIs · SPPS · Sustainable processes

\section{Introduction}

The therapeutic asset of peptide-based APIs has significantly grown over the preceding decades and today about 80 peptide drugs are already marketed ensuring a bright future for peptide therapeutics. ${ }^{[1]}$ However unfavorable pharmacokinetics and limited bioavailability are major challenges in peptide drug development. These are continuously addressed by diverse research efforts including novel formulation and delivery techniques as well as designing stable analogs of peptide drugs. ${ }^{[2,3]}$ Whilst being well established, the synthesis and purification technologies are an area for which innovation and development of sustainable and robust processes in the production of peptide APIs is sought after. ${ }^{[4]}$ For the last five decades, Bachem has been a highly reliable provider of high-quality peptide APIs manufactured under cGMP (current good manufacturing practices) standards and therefore a trusted partner for numerous biopharmaceutical companies in their peptide drug development programs. ${ }^{[5]}$ Our commitment and capabilities to provide access to high-quality peptide APIs on a large-scale have been well demonstrated. As a case study, the production of Octreotide on kilograms batch scale with more than $99.9 \%$ purity is briefly discussed here.

At Bachem we are continuously striving to improve our state-of-the-art synthetic and purification platform as well as to invest resources into scouting new technologies and effectively implementing them to minimize environmental impact of largescale API production. In this context, Bachem has systematically developed a strategy to identify and evaluate environmentally friendly solvent alternatives for solid-phase peptide synthesis (SPPS). How this objective is achieved without changing overall the holistic approach of the current state-of-the-art SPPS is further discussed here. In addition, a completely new platform for peptide synthesis, the Molecular Hiving ${ }^{\mathrm{TM}}$ technology, that has been developed by Jitsubo and was recently established at Bachem, is also highlighted. Molecular Hiving ${ }^{\mathrm{TM}}$ is a combination of best-ofboth-worlds i.e. SPPS and liquid-phase peptide synthesis (LPPS).

${ }^{*}$ Correspondence: : Dr. R. Schönleber, E-mail: ralph.schoenleber@bachem.com Bachem AG, Hauptstrasse 144, CH-4416 Bubendorf, Switzerland
To further highlight Bachem's innovative strength in the solventconsuming downstream processes, MCSGP (multi-column countercurrent solvent-gradient purification) as future technology for peptide purification is briefly introduced. The MCSGP technology allows substantial reduction in process mass intensity (PMI), which is inherently associated with traditional HPLC purification of peptides. Taken together, this review highlights the efforts and results which have been driven by our passion for innovation at Bachem to make the entire production process more sustainable and eco-friendly while keeping our utmost commitment to producing high-quality peptide APIs.

\section{Large-scale Production of Peptide APIs: A Case Study of Octreotide}

Production of peptides on large scale requires robust and efficient synthetic technologies, a skilled workforce, and strict process control.[6,7] Bachem's capacity to synthesize peptides on such a large-scale as well as its efficient downstream processing to achieve production of the final product on a multiple kilogram scale has been demonstrated. Octreotide is a widely known analog of naturally occurring somatostatin used as drug against diverse indications including acute hemorrhage. ${ }^{[8]}$ This cyclic octapeptide D-Phe-cyclo[Cys-Phe-D-Trp-Lys-Thr-Cys]-Thr(ol) is one of the generic APIs in Bachem's portfolio. The manufacturing process for highly pure (>99.9\%) Octreotide acetate was systematically and successfully established on an 18 kilogram batch size. The five important stages achieving this target are depicted in Fig. 1, which includes large-scale Fmoc-SPPS, acidic cleavage of synthesized peptide from solid support and its precipitation and isolation in the crude form, downstream purification via HPLC, ion exchange (from trifluoroacetate to acetate salt) of purified peptide and finally isolation of high-quality peptide via freeze drying. The large scale SPPS (24 mol scale with $30-50 \mathrm{~kg}$ of starting resin) was achieved by employing a 1000 L SPPS-reactor and was optimized to obtain linear Octreotide with crude purity of approx. $86 \%$. After cyclization of crude, linear Octreotide, the use of a $59 \mathrm{~cm}$ column for preparative RP-HPLC enabled purification of up to $3 \mathrm{~kg}$ crude cyclic peptide in a single run. After purification, Octreotide is isolated as trifluoroacetate salt in $65 \%$ yield (for oxidation and purification) with $>99.9 \%$ purity. In the final 
process step, Octreotide is converted into the acetate salt (99\% yield) and isolated by lyophilization. The large-scale manufacture of highly pure (>99.9\%) Octreotide required a well developed, strictly controlled protocol minimizing the impurities originating from different sources such as starting materials, SPPS-related processes, and instability of the peptide. Additionally, the overall yield of approx. $60 \%$ for the large-scale manufacturing process of Octreotide proves remarkable progress. We envisage that using innovative purification methods such as multi-column countercurrent solvent-gradient purification (MCSGP) might be an innovative approach for further increase of purification yields and overall process efficiency.

The use of closed trays for the final freeze-drying process in peptide API manufacture is a breakthrough Bachem innovation. ${ }^{9]}$ This technology provides two additional advantages, the closedsystem avoids last-stage contamination during freeze-drying and improves occupational safety with the additional barrier protection. Bachem is committed to protecting the environment from the chemical footprint and the safety of the community, therefore we recalibrate our resources and technologies constantly on a scale that should help to minimize the impact of upstream and downstream processes on the environment. Our efforts of greening peptide chemistry are globally recognized. The US Environment Protection Agency (US EPA) has jointly honored Bachem and Amgen Inc. with the 2017 Green Chemistry Challenge Award for developing an innovative green process for the commercial manufacture of Etelcalcetide (API in Parsabiv ${ }^{\mathrm{TM}}$ ). ${ }^{[10]}$ The improved technology has reduced $71 \%$ of solvent consumption, $56 \%$ of operating time, and $76 \%$ of manufacturing cost while increasing manufacturing capacity by five-fold. Further insights into the greener approach taken at Bachem are discussed below.

\section{3. 'Green' Alternative Solvents in Fmoc-SPPS}

$N, N$-Dimethylformamide (DMF) is a commonly used solvent in SPPS both in academia and industries. ${ }^{[11]}$ DMF is the gold standard solvent mainly because of its physical properties such as polarity and viscosity which universally suit it to the essential parameters of SPPS such as optimal resin swelling, reagent solubility, efficient coupling and Fmoc-removal reactions. However, DMF is categorized as a substance of very high concern (SVHC) by the European Chemical Agency (ECHA) due to its environmental and health hazards. ${ }^{[12]}$ Therefore there is an urgent need to identify an environmentally friendly alternative to DMF in SPPS. A lot of efforts have been provided by academia as well as industry to find a greener alternative to DMF. ${ }^{[13]}$ Recently, Bachem has led with focused resources a study in collaboration with Novo Nordisk to identify green and viable solvent alternatives as a replacement for DMF in state-of-theart SPPS technology. ${ }^{[14-16]}$ The investigations afforded important insights into harnessing polarity and viscosity of solvents for identification of viable alternatives to DMF. Selected solvents were screened against a series of parameters essential for effective SPPS such as swelling of PS resin (starting and peptidyl resins), solubility of amino acid derivatives, reaction kinetics of coupling and Fmoc-removal steps, inertness of solvents (stability of solvents) as well as their impact on classical side reactions in SPPS. These experiments generated a toolbox of solvents that can be used to replace DMF in SPPS in the future. Particularly, for the replacement of DMF in PS-resin-based Fmoc-SPPS at room temperature without making drastic changes in the contemporary state-of-the-art of SPPS technology, it is of key importance to identify a solvent that shows equivalent polarity and viscosity to DMF at room temperature. In our findings, we could not identify any single solvent among the screened solvents that shows in its neat form a similar polarity-viscosity (PV) profile as DMF. However, mixing two neat solvents in an appropriate proportion allowed to swiftly fine-tune the PV properties of the solvent mixture close to the PV properties of DMF. As shown in Fig. 2, it is possible to fine-tune the composition of a binary mixture of dimethyl sulfoxide (DMSO)/1,3-dioxolane (DOL), DMSO/2-methyl tetrahydrofuran (2-Me-THF) and DMSO/ethyl acetate (EtOAc) close to DMF on the PV plot. These binary mixtures are anticipated as viable alternatives to DMF in SPPS. The 3:7 compositions of these binary mixtures occupy spaces close to DMF on the PV plot. On the other hand, it was not possible to obtain any combinations from $N$-butyl pyrrolidinone (NBP) or $N$-formyl morpholine (NFM) with other green solvents (such as EtOAc, DOL, 2-Me-THF) with a PV profile similar to DMF. Several fully automated and DMF-free test syntheses of Bivalirudin were performed explicitly in selected binary solvents and results were compared with a reference synthesis in DMF (data not shown) ${ }^{[15]}$ which suggest that apart from being a viable alternative to DMF, binary solvents are also beneficial to mitigate common side-reactions in SPPS.
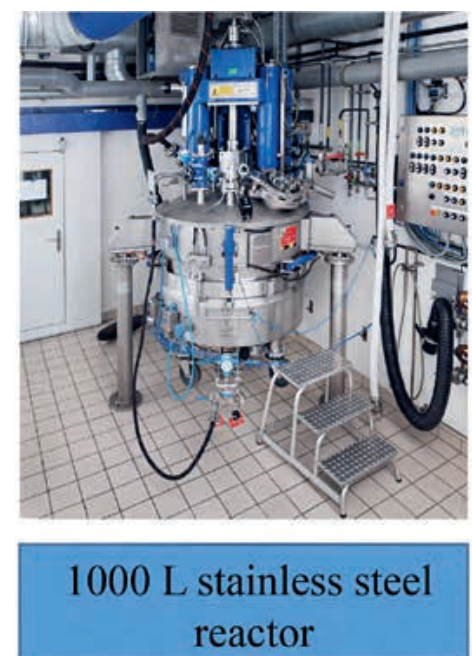

1. Fmoc-SPPS
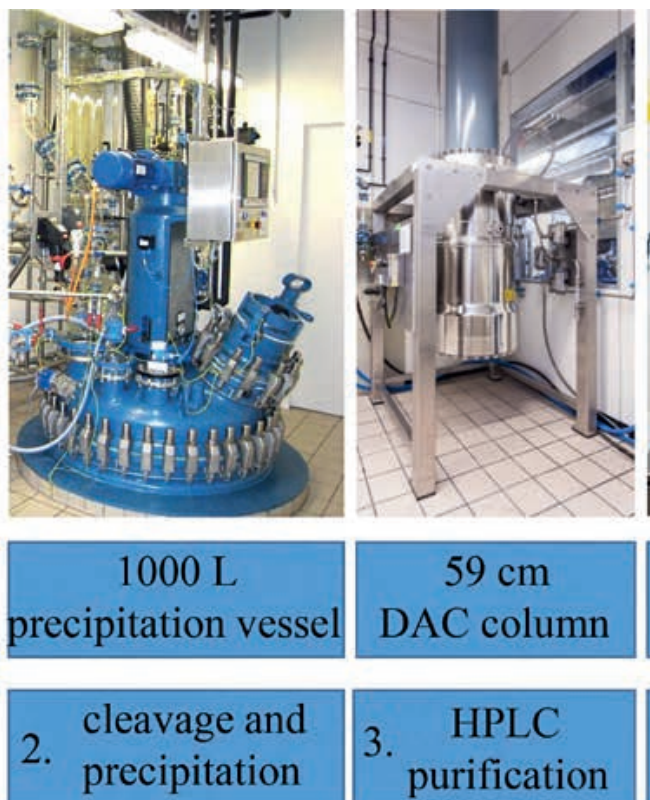
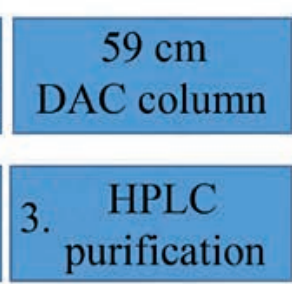

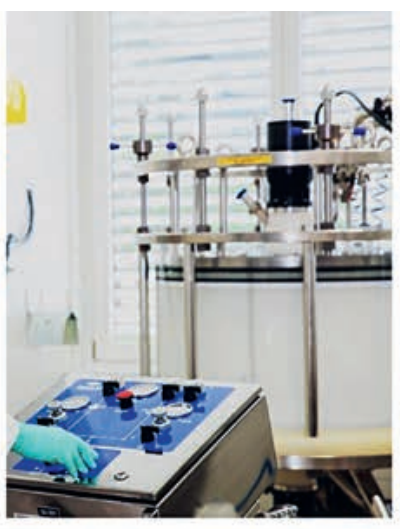

LPLC column and skid

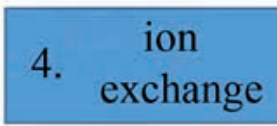

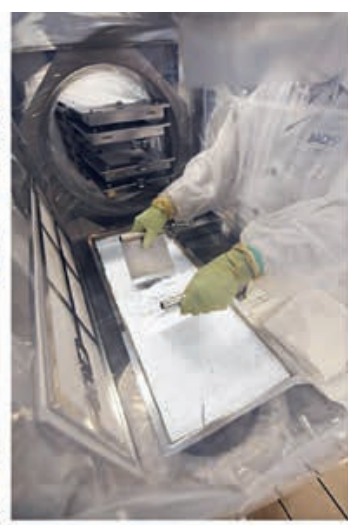

freeze drying in clean room (ISO 8)

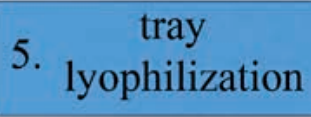

Fig. 1. Sophisticated equipment involved in various stages of large-scale production of Octreotide. 


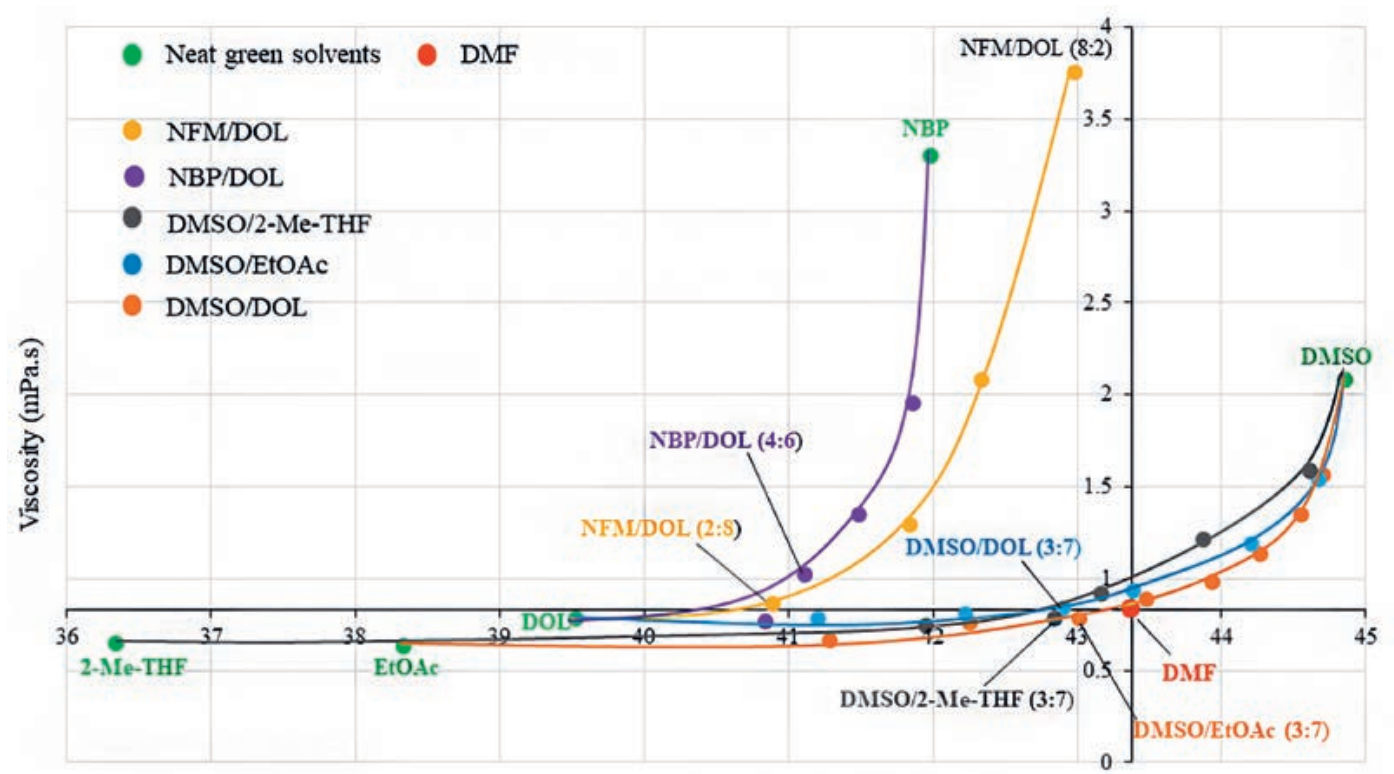

Polarity (Reichardt scale, $\mathrm{E}_{\mathrm{T}} 30 \mathrm{kcal} / \mathrm{mol}$ )
Fig. 2. Polarity-viscosity plot (PV plot) depicting the swift modulation of key physical properties of individual green solvents via recruiting them as a binary mixture. The neat green solvents are highlighted in green color and DMF as reference is highlighted in red (at origin). The line fitting is illustrative and not a regression fitting.

\section{Molecular Hiving ${ }^{\mathrm{TM}}$ Technology}

Finding an alternative solvent to DMF for traditional FmocSPPS is a daunting task with the main challenge for an alternative solvent to swell the polymeric resin to an appropriate extent while ensuring uniform diffusion throughout the resin bed. A completely different approach, the Molecular Hiving ${ }^{\mathrm{TM}}$ technology is an attractive alternative to Fmoc-SPPS for manufacturing short to medium-sized peptide APIs. ${ }^{[17]}$ Unlike the heterogeneous SPPS method where resin-beads remain insoluble throughout the synthesis, this homogeneous soluble tag-based technology does not require resin swelling and solvent diffusion. Molecular Hiving ${ }^{\mathrm{TM}}$ therefore offers a broad space for environmentally friendly solvent selection. Bachem has an exclusive license agreement with Jitsubo CO. LTD. for its Molecular Hiving ${ }^{\mathrm{TM}}$ technology. ${ }^{[18]}$ The working principle of Molecular Hiving ${ }^{\mathrm{TM}}$ technology is outlined in Fig. 3 and combines the benefits from LPPS and SPPS. The highlights of this synergy platform are

i. the use of a soluble tag molecule to anchor the growing peptide chain,

ii. amino acid derivatives that are used in classical Fmoc/tBuSPPS can be directly recruited in this process in much lesser excess,

iii. it does not require isolation of intermediates,

iv. in-process control (IPC) is readily achieved, v. overall solvent consumption is significantly reduced, up to 4-fold and, more importantly,

vi. Molecular Hiving ${ }^{\mathrm{TM}}$ technology allows the convenient choice of solvents and reagents which are free from carcinogenic, mutagenic and reprotoxic (CMR) substances.

Overall, the Molecular Hiving ${ }^{\mathrm{TM}}$ technology exemplifies that apart from benefitting the environment, approaches for 'green'ing traditional processes and finding innovative platforms also benefits the productivity and the cost-efficiency of processes (less solvent consumption, less excess of reagents, inexpensive tag molecules compared to polymeric resin, etc.).

\section{Multi-column Countercurrent Solvent-Gradient Purification (MCSGP) Technology}

The purification step is one of the most critical steps in the overall peptide API manufacturing process. It is required to ensure high-quality API supply for drug development. Reversed-phase (RP) or ion-exchange (IEX)-HPLC are traditional methods of purification that mainly suffer from compromised yield and productivity while obtaining high-purity of APIs. Moreover, a large solvent consumption is inherently associated with this batch-mode downstream process. The novel purification technique MCSGP (multi-column countercurrent solvent-gradient purification) is an innovative approach that allows internal recycling of overlapped

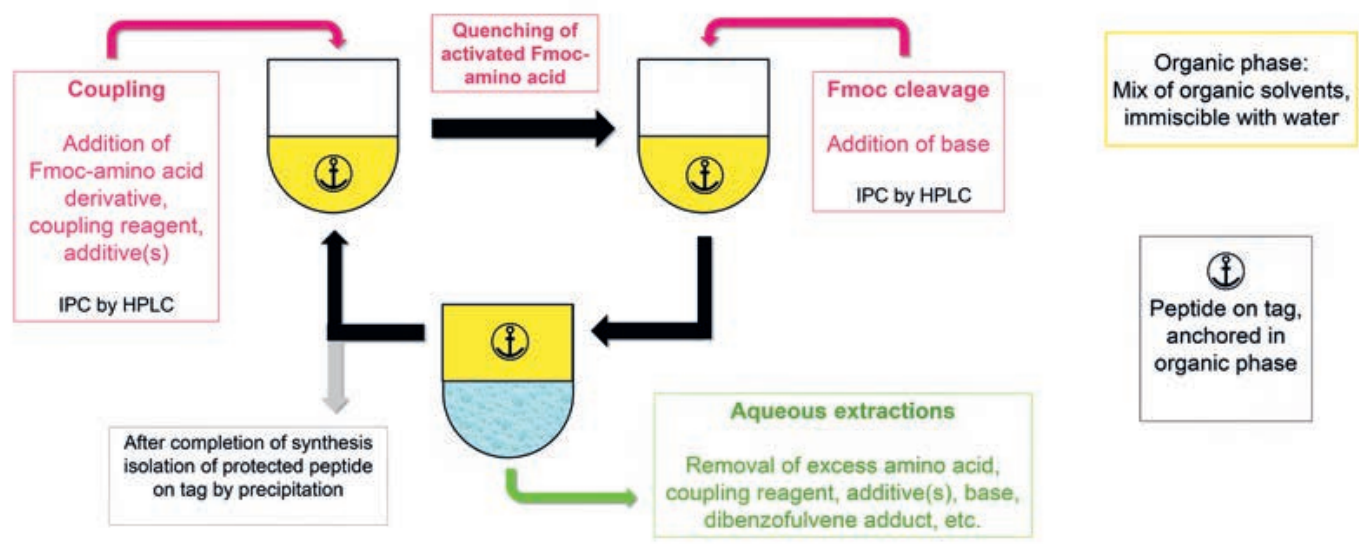

Fig. 3. Illustration of the principle of Molecular Hiving ${ }^{\mathrm{TM}}$ technology. 


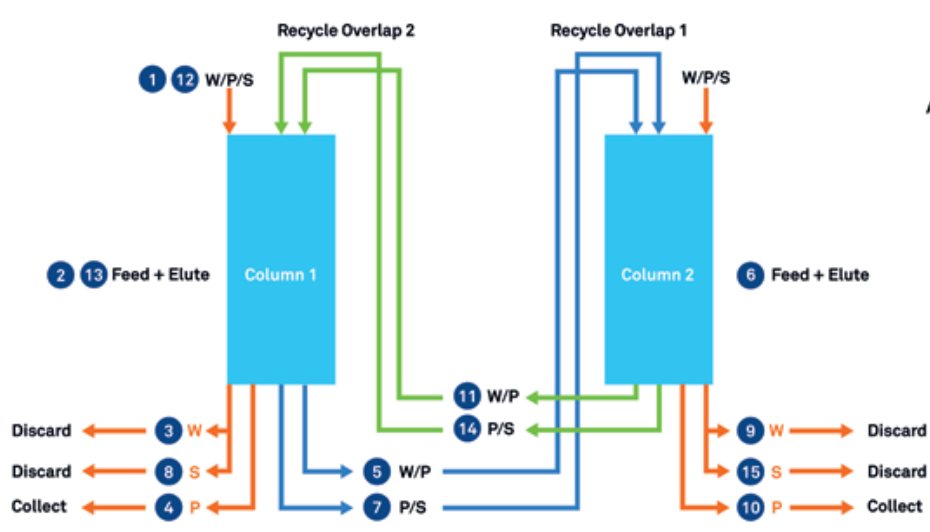

Fig. 4. Schematic illustration of the MCSGP principle. The regulation of sequential tasks (1-15, blue circles) on two columns is shown on the left. A representation of overlapping fractions containing the pure product $(P)$, weakly-adsorbing impurity (W), and strongly-adsorbing impurity (S) is shown on the right side. fractions that are continuously subjected to re-purification on an additional column placed in series ${ }^{[19]}$ Such continuous chromatography has been proven to increase yield by 4 -fold, productivity by 10 -fold, and to decrease solvent consumption by $70 \%$ without compromising the final purity of an API compared to the batch purification method. [20,21] Bachem is adopting MCSGP innovation with potential improvements in purification methods enabling the efficient and commercial benefits in the manufacturing of highly pure APIs. The working principle for a two-column MCSGP process is depicted in Fig. 4. The tasks (1-15) are computed and fully automated for a given purification cycle while at the end of each cycle the positions of two columns are exchanged via a simulated valve-switch.

The MCSGP process has prominent advantages over single-column batch chromatography such as higher yield, lower solvent consumption (up to $70 \%$ reduction) and lowered PMI which substantially reduces the chemical footprint on the environment. This technology once again underlines that while benefitting the environment, the 'green'ing of chemical processes also improves the time and cost-efficiency of the manufacturing processes.

\section{Conclusion}

Albert Einstein once said, "Life is like riding a bicycle. To keep your balance, you must keep moving". Since its foundation by Peter Grogg in 1971, Bachem is marching ahead as a leading player in the niche peptide API market for the last 50 years. Our innovative approaches and openness towards continuous improvements are guiding us to keep a critical balance between increasing market demands for large-scale manufacture of APIs and the impact of involved chemical and purification processes on the environment. To minimize the negative effect of chemical processes on the environment, we have joined forces with our collaborative partners not only to make the current manufacturing state-of-the-art technologies more sustainable, but also to identify new technologies with the intrinsic potential of minimal environmental footprint. As we look forward, while keeping our prime commitment to supply large-scale high-quality peptide APIs, our resolution to innovate and implement novel and environment-friendly technologies will pave the way for a sustainable future. We have also learnt that eco-friendly transformation of the manufacturing processes is not only advantageous for the environment but also makes the overall process more time-efficient and cost-effective.

\section{Acknowledgments}

We are celebrating the golden jubilee year 2021 of Bachem. Over the 50 years, numerous colleagues have contributed to the success of Bachem. We would like to express our appreciation and gratitude to all of them. Moreover, we would like to thank our collaborating partners as well as customers.

Received: March 31, 2021
[1] M. Muttenthaler, G. F. King, D. J. Adams, P. F. Alewood, Nat. Rev. Drug Discov. 2021, 20, 309, https://doi.org/10.1038/s41573-020-00135-8.

[2] A. Henninot, J. C. Collins, J. M. Nuss, J. Med. Chem. 2018, 61, 1382 , https://doi.org/10.1021/acs.jmedchem.7b00318

[3] K. A. Witt, T. J. Gillespie, J. D. Huber, R. D. Egleton, T. P. Davis, Peptides 2001, 22, 2329, https://doi.org/10.1016/s0196-9781(01)00537-x

[4] A. Isidro-Llobet, M. N. Kenworthy, S. Mukherjee, M. E. Kopach, K. Wegner, F. Gallou, A. G. Smith, F. Roschangar, J. Org. Chem. 2019, 84, 4615, https://doi.org/10.1021/acs.joc.8b03001.

[5] M. Mergler, G. Loidl, M. Diekmann, F. Dick, Chimia 2013, 67, 874, https://doi.org/10.2533/chimia.2013.874

[6] B. L. Bray, Nat. Rev. Drug Discov. 2003, 2, 587 , https://doi.org/10.1038/nrd1133

[7] T. Bruckdorfer, O. Marder, F. Albericio, Curr. Pharm. Biotechnol. 2005, 5 , 29, https://doi.org/10.2174/1389201043489620.

[8] https://www.drugs.com/monograph/octreotide.html.

[9] A. Müller, C. Erdin, WO 2017/137637, 2017.

[10] https://www.epa.gov/greenchemistry/green-chemistry-challenge2017-greener-reaction-conditions-award.

[11] R. Behrendt, P. White, J. Offer, J. Pept. Sci. 2016, 22, 4, https://doi.org/10.1002/psc.2836.

[12] https://echa.europa.eu/de/substance-information/-/substanceinfo/100.000.617.

[13] O. Al Musaimi, B. G. De La Torre, F. Albericio, Green Chem. 2020, 22, 996, https://doi.org/10.1039/c9gc03982a.

[14] V. Martin, S. Jadhav, P. H. G. Egelund, R. Liffert, H. Johansson Castro, T. Krüger, K. F. Haselmann, S. T. Le Quement, F. Albericio, F. Dettner, C. Lechner, R. Schönleber, D. S. Pedersen, Green Chem. 2021, 23, 3295, https://doi.org/10.1039/D1GC00603G.

[15] S. Jadhav, V. Martin, P. H. G. Egelund, H. Johansson Castro, T. Krüger, F. Richner, S. T. Le Quement, F. Albericio, F. Dettner, C. Lechner, R. Schönleber, D. S. Pedersen, Green Chem. 2021, 23, 3312, https://doi.org/10.1039/D1GC00604E.

[16] P. H. G. Egelund, S. Jadhav, V. Martin, H. Johansson Castro, F. Richner, S. T. Le Quement, F. Dettner, C. Lechner, R. Schönleber, D. S. Pedersen, 2021, submitted.

[17] https://www.jitsubo.com/en/publications/.

[18] https://www.bachem.com/news-media/news/news/article/bachem-and-jitsubo-enter-into-exclusive-licensing-agreement.

[19] L. Aumann, M. Morbidelli, WO/2006/116886, 2006.

[20] T. Müller-Späth, G. Ströhlein, O. Lyngberg, D. Maclean, Chim. Oggi/ Chemistry Today 2013, 31, 56

[21] T. Müller-Späth, M. Bavand, Pharm. Eng. March/April, 2019.

\section{License and Terms}

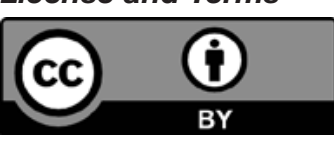

This is an Open Access article under the terms of the Creative Commons Attribution License CC BY 4.0. The material may not be used for commercial purposes.

The license is subject to the CHIMIA terms and conditions: (http:// chimia.ch/component/sppagebuilder/?view=page \&id=12).

The definitive version of this article is the electronic one that can be found at https://doi.org/10.2533/chimia.2021.476 